\title{
GLOBAL ATTRACTIVITY IN TIME-DELAYED PREDATOR-PREY SYSTEMS
}

\author{
YULIN $\mathrm{CAO}^{1}$ and $\mathrm{H}$. I. FREEDMAN ${ }^{2}$
}

(Received 26 May 1994; revised 5 October 1994)

\begin{abstract}
For a predator-prey model with time-delay due to gestation, criteria are obtained for persistence and global attractivity. The global attractivity criteria apply only to models with a decreasing prey isocline.
\end{abstract}

\section{Introduction}

Many known biological processes involve a time delay. In particular, in the mathematical modelling of population ecological interactions, time delay models have come into prominence. The recent seminal text of Y. Kuang [18] and the plethora of references contained therein show this to be the case.

Time delay models are significantly more difficult to analyze than instantaneous continuous models. Hence, although the existence of equilibria for ordinary differential equations and time-delayed equations are equivalent, their stability analyses are more difficult and questions of global stability are extremely more difficult in the later case. As well, the questions of persistence (to be discussed in Section 3) is also more difficult.

The question of global stability of equilibria is of particular interest to wildlife managers. If it is known that a system exhibits such global stability, then ecological planning based on a fixed eventual population can be carried out. For single species models [6], [9], [18] and competition models [14], [18], criteria have been developed to obtain global stability. However in the case of a predator-prey system, felt by many

\footnotetext{
'Department of Mathematics, University of Georgia, Athens, Georgia 30602, USA.

Present address: Department of Mathematics, SUNY at Farmingdale, Route 110, Farmingdale, NY 11735-1021, USA

${ }^{2}$ Applied Mathematics Institute, Department of Mathematics, University of Alberta, Edmonton, Alberta T6G 2G1, Canada

(C) Australian Mathematical Society, 1996, Serial-fee code 0334-2700/96
} 
to be the most interesting of ecological interactions, although there exist criteria for global stability in predator-prey models with no delays [7], [8], [17], [19] and criteria for local stability in predator-prey models with delays [11], there are as yet to the best of our knowledge no published criteria for global stability in predator-prey models with delays.

In models with complicated dynamics, the questions of persistence and extinction are important and fundamental. The definitions will be reviewed in Section 3. In this case criteria have been established for delay-differential equations in general [12], [16] and in particular for population models [3], [4], [5], [18], [20]. Here we obtain new criteria for our system.

The paper is organized as follows. In Section 2 we describe our model and some of its properties. In Section 3 the persistence-extinction question is discussed. This is followed by the question of global attractivity in Section 4. In Section 5 we illustrate our result with an example, and a brief discussion concludes the paper in Section 6 .

\section{The model}

In [11], a predator-prey model was described incorporating a time delay due to gestation in the predator equation. Here we will consider a general class of such models which contains the model described in [11] as a special case. (We will compare our results with those of [11] in Section 5.)

Hence we wish to consider the system

$$
\begin{aligned}
\dot{x}(t) & =f(x(t), y(t)) \\
\dot{y}(t) & =g(x(t-\tau), y(t)),
\end{aligned}
$$

subject to the initial conditions

$$
x(\theta)=x_{0}(\theta) \geq 0, \quad y(0)=y_{0} \geq 0,
$$

where $\tau>0$ is a constant time delay and $x_{0}(\theta)$ is a continuous function from $[-\tau, 0]$ to $R . x(t)$ and $y(t)$ represent the biomass of prey and predators, respectively at time $t \geq 0$.

In hypotheses (H1)-(H3) below, we outline the general assumptions to make system (2.1) model a predator-prey system with delay due to gestation:

(H1): $f(x, y)$ and $g(x, y)$ are sufficiently smooth so that solutions to (2.1)-(2.2) exist, are unique and are continuable for all positive time (see [15]),

(H2): $f(0, y)=g(x, 0)=0$ for all $x, y \geq 0, \lim _{x \rightarrow 0^{+}} x^{-1} f(x, 0)>0, \lim _{y \rightarrow 0^{+}} y^{-1} g(0, y)<0$,

(H3): $\partial f(x, y) / \partial y<0, \partial g\left(x_{\tau}, y\right) / \partial x_{\tau}>0, x, x_{\tau}, y \geq 0$, where we have used the notation 


$$
x_{\tau}(t) \triangleq x(t-\tau)
$$

For the purpose of our analyses, we will require $(\mathrm{H} 4)$ or $(\mathrm{H} 4)^{\prime}$ to be satisfied.

(H4): There exist two continuous functions $\varphi(y)$ and $\psi(x)$ from $[0,+\infty)$ to $\mathbb{R}$ with $\varphi(0)=k$ for some $k>0$ such that

$$
f(x, y)(x-\varphi(y))<0, \text { for all } x>0, y \geq 0, x \neq \varphi(y)
$$

and

$$
g(x, y)(y-\psi(x))<0, \text { for all } x \geq 0, y>0, y \neq \psi(x) .
$$

The next hypothesis is more general than (H4) and is only used for uniform persistence. (H4)' There exists a $K>0$ such that

$$
f(x, y)<0 \text { for all } x>K, y \geq 0,
$$

and for any $x_{1} \geq 0$ there exists $y_{*}=y_{*}\left(x_{1}\right) \geq 0$ such that

$$
g(x, y)<0 \text { for all } x \in\left[0, x_{1}\right], y>y_{*} .
$$

Notice that if (H4) is satisfied then $x=\varphi(y)$ and $y=\psi(x)$ are isoclines for (2.1) and any equilibrium of (2.1) is determined by solving the equations $x=\varphi(y)$ and $y=\psi(x)$. One can see that under assumptions (H1)-(H3), for any given initial condition (2.2) with $x_{0}(0)>0$ and $y_{0}>0$, the solution to (2.1) and (2.2) satisfies $x(t)>0$ and $y(t)>0$ for all $t \geq 0$. We now establish the following relation between (H4) and (H4)'.

Proposition 2.1. Let $(H 4)$ be satisfied. Then $(H 4)^{\prime}$ is also satisfied with $y_{*}\left(x_{1}\right)=$ $\max _{x \in\left[0, x_{1}\right]}\{\psi(x)\}$.

PROOF. If $y=0$ in (2.4), then $f(x, 0)(x-K)<0$ for all $x>0$ with $x \neq K$. Thus $f(x, 0)<0$ for all $x>K$. Since $\frac{\partial f}{\partial y}(x, y) \leq 0$ for all $x \geq 0$ and $y \geq 0$, it follows that

$$
f(x, y) \leq f(x, 0)<0 \text { for all } x>0 \text { and } y>0 .
$$

Now, let $y_{*}=\max \left\{\psi(x): x \in\left[0, x_{1}\right]\right\}$. For any $x \in\left[0, x_{1}\right]$ and $y>y_{*}$, we have $y>\psi(x)$. Consequently (2.5) implies that $g(x, y)<0$ for all $x \in\left[0, x_{1}\right]$ and $y>y_{*}$. 
We conclude this section by a discussion of the significance of (H4). If (H4) is satisfied then the prey isocline is given by $x=\varphi(y)$ and the predator isocline by $y=\psi(x)$. Hence (2.4) can only be satisfied if the prey isocline is strictly decreasing. This would be true if system (2.1) is a Lotka-Volterra system [8] or if for example $f(x, y)=r x(1-x / K)-a x y /(b+x)$ and the isocline given by $y=(r / a)(1-x / K)(b+x)$, which is a parabola, is such that the vertex occurs for $x<0$. However, in general the prey isocline is not decreasing for all $x>0$, and so (H4) can only be satisfied for a restricted class of predator-prey models.

\section{Uniform persistence}

In this section we show that under our assumptions, system (2.1)-(2.2) with positive initial conditions is uniformly persistent.

We take our definition of uniform persistence from [13]. For the definition of uniform persistence in a more general setting see [1], [2], [10]. We say that our system (2.1)-(2.2) exhibits uniform persistence provided the solution $(x(t), y(t))$ to (2.1)-(2.2) satisfies $x(t)>0, y(t)>0, t \geq 0$ and there exists $\delta>0$ independent of solutions of (2.1)-(2.2) such that $\liminf _{t \rightarrow \infty} x(t) \geq \delta$ and $\liminf _{t \rightarrow \infty} y(t) \geq \delta$.

LEMMA 3.1. Suppose (HI)-(H3) and (H4)' are satisfied. If $(x(t), y(t))$ is a solution of (2.1)-(2.2) with $x_{0}(0) \geq 0$ and $y_{0} \geq 0$, then

$$
\limsup _{t \rightarrow+\infty} x(t) \leq K
$$

and

$$
\limsup _{t \rightarrow+\infty} y(t) \leq y_{*}(K),
$$

where $K$ and $y_{*}($.$) are given by (H 4)^{\prime}$.

PROOF. We let $\hat{x}=\max _{t \in[-\tau, 0]}\{x(t)\}$ and $\hat{x}_{0}=\max \{\hat{x}, K\}$. Since $f(x, y)<0$ for all $x>K$ and $y \geq 0$, it is easy to show that

$$
0 \leq x(t) \leq \hat{x}_{0}
$$

for all $t \geq 0$. Similarly since $g(x, y)<0$ for all $0 \leq x \leq \hat{x}_{0}$ and $y>y_{*}\left(\hat{x}_{0}\right)$, we can show that

$$
0 \leq y(t) \leq \max \left\{y(0), y_{*}\left(\hat{x}_{0}\right)\right\}
$$


Since $y(t)$ is bounded, it follows from (2.6) that

$$
\limsup _{t \rightarrow+\infty} x(t) \leq K
$$

Consequently it follows from (2.7) that

$$
\limsup _{t \rightarrow+\infty} y(t) \leq y_{*}(K) \text {. }
$$

We now prove our result on uniform persistence. Some additional conditions are needed so that the boundary equilibria of system (2.1)-(2.2) do not attract any positive solutions of $(2.1)-(2.2)$.

THEOREM 3.2. Suppose that $(H 1)-(H 3)$ and $(H 4)^{\prime}$ are satisfied. Assume that there exists $K_{0}>0$ such that $f(x, 0)\left(x-K_{0}\right)<0$ for all $x>0$ with $x \neq K_{0}$. If $g(0, y)<0$ for all $y>0, f(x, y)>0$ for $x>0$ and $y \geq 0$ sufficiently small, and $g\left(K_{0}, 0\right)>0$, then system (2.1)-(2.2) is uniformly persistent.

PROOF. By Lemma 3.1, we know that system (2.1)-(2.2) is dissipative. We wish to use the technique developed in [1], termed BFW, to show the uniform persistence. Consider system (2.1)-(2.2) defined on the boundary $x=0$ or $y=0$. If $y=0$, then (2.1) becomes

$$
\dot{x}(t)=f(x(t), 0)
$$

This ordinary differential equation has two equilibria $x=0$ and $x=K_{0}$, where the second one attracts all positive solutions of (3.3). If $x=0$, then (2.1) becomes

$$
\dot{y}(t)=g(0, y(t)) .
$$

Since $g(0, y)<0$ for all $y>0$, every positive solution $y$ of (3.4) satisfies $\lim _{t \rightarrow+\infty} y(t)=$ 0 . Thus, there are only two boundary equilibria, $E_{0}(0,0)$ and $E_{1}\left(K_{0}, 0\right)$ for $(2.1)$. The condition $g\left(K_{0}, 0\right)>0$ implies that the equilibrium $E_{1}$ does not attract any positive solution of (2.1), that is, there is no positive solution $(x(t), y(t))$ of (2.1) satisfying $\lim _{t \rightarrow+\infty}(x(t), y(t))=\left(K_{0}, 0\right)$. Since $f(x, y)>0$ for $0<x<<1$ and $0 \leq y<<1$, there is no positive solution of (2.1) approaching $E_{0}(0,0)$. Consequently, by applying the results contained in the main theorem of [16], system (2.1) is uniformly persistent.

The followng corollary gives related results. 
COROLLARY 3.3. Suppose (HI)-(H4) are satisfied. If $\psi(0) \leq 0$ and $\psi(K)>0$, where $K$ is defined in (H4), then system (2.1)-(2.2) is uniformly persistent. In particular, any positive solution $(x(t), y(t))$ of $(2.1)-(2.2)$ satisfies

$$
\limsup _{t \rightarrow+\infty} x(t) \leq K
$$

and

$$
\limsup _{t \rightarrow+\infty} y(t) \leq \max _{x \in[0, K]}\{\psi(x)\} .
$$

PROOF. Since it follows from Proposition 2.1 that (H4) implies (H4)', it then follows from (2.4) that $\psi(0) \leq 0$ implies $y(0, y)<0$ for all $y>0$ and $\psi(K)>0$ implies $g(K, 0)>0$, where $K_{0}$ in Theorem 3.2 equals $K$ in this case. Finally, the condition $\varphi(0)=K>0$ implies that $f(x, y)>0$ for $0<x<<1$ and $0 \leq y<<1$. Therefore, all conditions in Theorem 3.2 are satisfied and consequently, system (2.1)(2.2) is uniformly persistent. The inequalities in Corollary 3.3 follow from Lemma 3.1 .

\section{Global attractivity}

In this section we will show that under assumptions (H1)-(H4), system (2.1)-(2.2) contains a globally asymptotically stable equilibrium. We remind the readers that hypothesis (H4) implies that there exists a strictly decreasing prey isocline, which is a substantial restriction. Nevertheless, this is the first result to be published showing global attractivity for predator-prey systems with time delay due to gestation.

Before stating and proving our theorem, we require the following lemma.

LEMMA 4.1. Suppose (H1)-(H4) are satisfied. Let $(x(t), y(t))$ be a positive solution of (2.1)-(2.2). Assume that there exist some positive constants $\delta_{0}, p_{0}, \mu_{0}$ and $q_{0}$ such that

$$
\delta_{0} \leq \liminf _{t \rightarrow+\infty} x(t) \leq \limsup _{t \rightarrow+\infty} x(t) \leq p_{0}
$$

and

$$
\mu_{0} \leq \liminf _{t \rightarrow+\infty} y(t) \leq \limsup _{t \rightarrow+\infty} y(t) \leq q_{0} .
$$

If $\tilde{q_{0}}$ and $\tilde{\mu_{0}}$ are respectively the maximum and minimum of $\psi(x)$ on the interval $\left[\delta_{0}, p_{0}\right]$ and $\tilde{\delta}_{0}$ and $\tilde{p}_{o}$ are respectively the maximum and minimum of $\varphi(y)$ on the interval $\left[\tilde{\mu_{0}}, \tilde{q_{0}}\right]$, then

$$
\tilde{\delta_{0}} \leq \liminf _{t \rightarrow+\infty} x(t) \leq \limsup _{t \rightarrow+\infty} x(t) \leq \tilde{p_{0}}
$$


and

$$
\tilde{\mu_{0}} \leq \liminf _{t \rightarrow+\infty} y(t) \leq \limsup _{t \rightarrow+\infty} y(t) \leq \tilde{q_{0}}
$$

PROOF. For any given $\epsilon>0$, it follows from the assumptions that there exists $T_{0}>0$ such that

$$
\delta_{0}-\epsilon \leq x(t) \leq p_{0}+\epsilon \quad \text { for all } t \geq T_{0}
$$

Consider the second equation of (2.1)

$$
\dot{y}(t)=g(x(t-\tau), y(t)) \quad \text { for } t \geq T_{0}+\tau .
$$

It follows from (H4) that, for $t \geq T_{0}+\tau$

$$
g(x(t-\tau), y)<0
$$

for $y>\max \left\{\psi(x): x \in\left[\delta_{0}-\epsilon, p_{0}+\epsilon\right]\right\}$, and

$$
g(x(t-\tau), y)>0
$$

for $0<y<\min \left\{\psi(x): x \in\left[\delta_{0}-\epsilon, p_{0}+\epsilon\right]\right\}$. Consequently, if

$$
\tilde{q}_{0}^{\epsilon}=\max \left\{\psi(x): x \in\left[\delta_{0}-\epsilon, p_{0}+\epsilon\right]\right\}
$$

and

$$
\tilde{\mu}_{0}^{\epsilon}=\min \left\{\psi(x): x \in\left[\delta_{0}-\epsilon, p_{0}+\epsilon\right]\right\},
$$

then it follows from (4.2) that

$$
\tilde{\mu}_{0}^{\epsilon} \leq \liminf _{t \rightarrow+\infty} y(t) \leq \limsup _{t \rightarrow+\infty} y(t) \leq \tilde{q}_{0}^{\epsilon} .
$$

Taking the limits above as $\epsilon \rightarrow 0^{+}$, we have proved (4.4), Now, for any $\epsilon>0$ there exists $T_{1} \geq 0$ such that

$$
\tilde{\mu}_{0}-\epsilon \leq y(t) \leq \tilde{q}_{0}+\epsilon \quad \text { for all } t \geq T_{1}
$$

Consider the first equation of (2.1)

$$
\dot{x}(t)=f(x(t), y(t)) \quad \text { for } t \geq T_{1} \text {. }
$$

It follows from (H4) that, when $t \geq T_{1}$,

$$
f(x, y(t))<0
$$


for $x>\max \left\{\varphi(y): y \in\left[\tilde{\mu}_{0}-\epsilon, \tilde{q}_{0}+\epsilon\right]\right\} \triangleq \tilde{p}_{0}^{\epsilon}$, and

$$
f(x, y(t))>0
$$

for $0<x<\min \left\{\varphi(y): y \in\left[\tilde{\mu}_{0}-\epsilon, \tilde{q}_{0}+\epsilon\right]\right\} \triangleq \tilde{\delta}_{0}^{\epsilon}$. In fact, for any $\left[x_{1}, x_{2}\right] \subset$ $\left(\tilde{p}_{0}^{\epsilon},+\infty\right)$ and $\left[\tilde{x}_{1}, \tilde{x}_{2}\right] \subset\left(0, \tilde{\delta}_{0}^{\epsilon}\right)$, there exists a constant $\delta>0$ such that $\tau \geq T_{1}$ and $x \in\left[x_{1}, x_{2}\right]$ imply

$$
f(x, y(t))>\delta
$$

and $t \geq T_{1}$ and $x \in\left[\tilde{x}_{1}, \tilde{x}_{2}\right]$ imply

$$
f(x, y(t))<-\delta \text {. }
$$

Consequently, as a solution of (4.10), $x(t)$ satisfies

$$
\tilde{\delta}_{0}^{\epsilon} \leq \liminf _{t \rightarrow+\infty} x(t) \leq \limsup _{t \rightarrow+\infty} x(t) \leq \tilde{p}_{0}^{\epsilon}
$$

Taking the limits above as $\epsilon \rightarrow 0^{+}$, we have verified (4.3).

In our next theorem we connect the global attractivity of the positive equilibrium for system (2.1) with the global attractivity of a fixed point for a specified mapping.

THEOREM 4.2. Suppose that $(H 1)-(H 4)$ are satisfied with $\varphi$ monotone decreasing and $\psi$ monotone increasing. Assume $\psi(0)=0$ and $\psi(K)>0$. Let $\sigma(x)=\varphi(\psi(x))$ be a mapping from $[0, K]$ to $\mathbb{B}$. If $\sigma((0, K]) \subset(0, K]$ and there exists a fixed point $x_{0} \in(0, K]$ of the mapping $\sigma$ which is globally attractive (that is, for any $x \in(0, K]$, $\sigma^{h}(x) \rightarrow x_{0}$ as $\left.h \rightarrow+\infty\right)$, then system (2.1)-(2.2) has a unique positive equilibrium $\left(x_{0}, \psi\left(x_{0}\right)\right)$ and this equilibrium attracts all positive solutions.

PROOF. First of all, it follows from the monotonicity of $\varphi$ and $\psi$ that the mapping $\sigma$ is monotone decreasing. By Corollary 3.3 one can see that system (2.1)-(2.2) is uniformly persistent. Consequently for any positive solution $(x(t), y(t))$ of (2.1)(2.2), there exist positive constants $\delta_{0}, p_{0}, \mu_{0}$ and $q_{0}$ with $p_{0} \leq K$ such that

$$
\delta_{0}=\liminf _{t \rightarrow+\infty} x(t) \leq \limsup _{t \rightarrow+\infty} x(t)=p_{0}
$$

and

$$
\mu_{0}=\liminf _{t \rightarrow+\infty} y(t) \leq \limsup _{t \rightarrow+\infty} y(t) \leq q_{0}
$$


It follows from Lemma 4.1 that

$$
\sigma\left(p_{0}\right) \leq \liminf _{t \rightarrow+\infty} x(t) \leq \limsup _{t \rightarrow+\infty} x(t) \leq \sigma\left(\delta_{0}\right)
$$

and

$$
\psi\left(\delta_{0}\right) \leq \liminf _{t \rightarrow+\infty} y(t) \leq \limsup _{t \rightarrow+\infty} y(t) \leq \psi\left(p_{0}\right)
$$

where $\sigma\left(p_{0}\right), \sigma\left(\delta_{0}\right), \psi\left(\delta_{0}\right)$ and $\psi\left(p_{0}\right)$ equal $\tilde{\mu_{0}}, \tilde{q_{0}}, \tilde{\delta_{0}}$ and $\tilde{p}_{0}$ in Lemma 4.1 respectively by the monotonicity of $\psi$ and $\sigma$.

Consequently, (4.16) and (4.18) yield

$$
\sigma\left(p_{0}\right) \leq \delta_{0} \leq p_{0} \leq \sigma\left(\delta_{0}\right)
$$

Since $p_{0} \leq K$ by Corollary (3.3), it follows from the assumption that $0<\sigma\left(p_{0}\right) \leq$ $\sigma\left(\delta_{0}\right) \leq K$. Therefore, by the assumption $\sigma((0, K]) \subset(0, K]$ and the monotonicity of $\sigma,(4.20)$ yields

$$
\sigma^{2}\left(\delta_{0}\right) \leq \sigma\left(p_{0}\right) \leq \sigma\left(\delta_{0}\right) \leq \sigma^{2}\left(p_{0}\right)
$$

Combining (4.20) and (4.21), we have

$$
\sigma^{2}\left(\delta_{0}\right) \leq \delta_{0} \leq p_{0} \leq \sigma^{2}\left(p_{0}\right) .
$$

It follows step by step that

$$
\sigma^{2 n}\left(\delta_{0}\right) \leq \delta_{0} \leq p_{0} \leq \sigma^{2 n}\left(p_{0}\right)
$$

for any positive integer $n$, where $\sigma^{k}$ is the $k$-th interaction of the mapping $\sigma$. Taking the limit of (4.23) as $n \rightarrow+\infty$, it follows from the global attractivity of $\sigma$ that

$$
\delta_{0}=p_{0}=x_{0}
$$

Consequently, (4.16) becomes $\lim _{t \rightarrow+\infty} x(t)=x_{0}$ and (4.19) becomes $\lim _{t \rightarrow+\infty} y(t)=\psi\left(x_{0}\right)$ for any positive solution $(x(t), y(t))$ of $(2.1)-(2.2)$.

REMARK 4.3. If in (H4) $\psi(0)<0$ and there exists $K^{\prime}<K$ such that $\psi\left(K^{\prime}\right)=0$, then in order to satsify the condition in Theorem 4.2 we can redefine $\psi(x) \equiv 0$ for $x \in\left[0, K^{\prime}\right]$. Certainly under this redefinition, (H4) is still valid. 
REMARK 4.4. Under the assumptions of Theorem 4.2, the positive equilibrium of system (2.1) is determined by solving the equations $x=\varphi(y)$ and $y=\psi(x)$ for $x$ and $y$. Consequently, the positive equilibrium of system (2.1) is determined by the fixed point of the mapping $\sigma$. Precisely, if $x_{0} \in[0, K]$ is a fixed point of the mapping $\sigma$, then $\left(x_{0}, \psi\left(x_{0}\right)\right)$ is an equilibrium of system (2.1). Of course, $\left(x_{0}, \psi\left(x_{0}\right)\right)$ is a positive equilibrium, since $x_{0}=K$ implies $\psi\left(x_{0}\right)>0$ and $x_{0} \in(0, K)$ implies the $\left(x_{0}, \psi\left(x_{0}\right)\right)$ is not a boundary equilibrium. Finally, since $\sigma(0)=K$ and $\sigma(K) \leq K$ there always exists a fixed point of $\sigma$ in $(0, K]$.

The following corollary gives a more easily computable criterion for global attractivity.

COROLlARY 4.5. Suppose the assumptions of Theorem 4.2 are satisfied except that the conditions on $\sigma$ are replaced by $\left|\sigma^{\prime}(x)\right|<1$ for all $x \in(0, K]$. Then there exists a unique positive equilibrium $\left(x_{0}, \psi\left(x_{0}\right)\right)$ of $(2.1)-(2.2)$ which attracts all positive solutions.

Proof. Since $\sigma(0)=K$, the assumption $\left|\sigma^{\prime}(x)\right|<1$ for all $x \in(0, K]$ and the monotonicity of $\sigma$ imply $\sigma((0, K]) \subset(0, K]$. By Remark 4.4, there always exists a fixed point $x_{0}$ in $(0, K]$ for the mapping $\sigma$, and this fixed point yields a positive equilibrium $\left(x_{0}, \psi\left(x_{0}\right)\right)$ for system (2.1)-(2.2). Since the fixed point $x_{0}$ of $\sigma$ is globally attractive, the proof now follows from Theorem 4.2.

\section{Application}

In this section, we will discuss an application of our results to a biological model considered in [11]. Consider the model

$$
\left\{\begin{array}{l}
\dot{x}(t)=x(t) g(x(t))-y^{m}(t) p(x(t)) \\
\dot{y}(t)=y(t)\left(-s+c y(t)^{m-1} p(x(t-\tau))-q(y(t))\right) .
\end{array}\right.
$$

This model prescribes predator-prey interactions with mutual interference, densitydependent predator death rate and time lag due to gestation. In (5.1), $x(t)$ represents prey density at time $t, y(t)$ represents predator density at time $t$ and $\tau$ is a constant time delay. The following conditions have been discussed and justified in [11]

(i) $g(0)>0, \quad \frac{d g}{d x} \leq 0$

(ii) $\exists K>0$ such that $g(K)=0$ and $g^{\prime}(K)<0$

(iii) $p(0)=0, \quad \frac{d p}{d x}>0$

(iv) $0<m<1$

(v) $q(0)=0, \quad \frac{d q}{d y} \geq 0$. 
(We add the condition $g^{\prime}(K)<0$ to ensure that the carrying capacity for the prey-only model is unique.)

The local stability of the positive equilibrium of system (2.1) was discussed in [11]. Here we obtain criteria for global attractivity.

Define

$$
\begin{aligned}
& H(x, y)=x \frac{d g(x)}{d x}+g(x)-y^{m} \frac{d p(x)}{d x} \\
& N(x, y)=-m y^{m-1} p(x)<0 \\
& Q(x, y)=c y^{m} \frac{d p(x)}{d x}>0
\end{aligned}
$$

and

$$
R(x, y)=(m-1) c y^{m-1} p(x)-y^{m} \frac{d p(y)}{d y} \leq 0 .
$$

THEOREM 5.1. Assume $H R>-N Q$ for all $0<y \leq\left(g(0) / p^{\prime}(0)\right)^{1 / m}$ and $0 \leq x \leq$ $K$. Then there exists a unique positive equilibrium for system (5.1) which is globally asymptotically stable.

Proof. Since $N(x, y)<0$ and $Q(x, y)>0$, the inequality $H R>-N Q$ implies $H(x, y)>0$ and $R(x, y)<0$ for $0 \leq x \leq K$ and $0<y \leq\left(g(0) / p^{\prime}(0)\right)^{1 / m}$. Therefore, one can solve $x=\varphi(y)$ from the equation

$$
x g(x)-y^{m} p(x)=0
$$

for $0 \leq x \leq K$ and $0 \leq y \leq\left(g(0) / p^{\prime}(0)\right)^{1 / m}$. It is easy to see that $\varphi(0)=K>0$, $\varphi(\hat{y})=0$ and $\varphi(y)$ is strictly decreasing for $y \in[0, \hat{y}]$, where we denote

$$
\hat{y}=\left(g(0) / p^{\prime}(0)\right)^{1 / m} \text {. }
$$

Similarly, one can solve $y=\psi(x)$ from the equation

$$
y\left(-s-q(y)-c y^{m-1} p(x)\right)=0
$$

for $0 \leq x \leq+\infty$ and $0 \leq y \leq \hat{y}$. It is easy to see that $\psi(0)=0$ and $\psi(x)$ is strictly increasing for $x \in[0,+\infty)$. Now, if we define $\varphi(y)=0$ for $y>\hat{y}$, then (H4) is satisfied. If $\sigma(x)=\varphi(\psi(x))$ then

$$
\begin{aligned}
\sigma^{\prime}(x) & =\varphi^{\prime}(\psi(x)) \psi^{\prime}(x) \\
& =-\frac{N(x, y)}{H(x, y)} \cdot \frac{-Q(x, y)}{R(x, y)}=\frac{N(x, y) Q(x, y)}{H(x, y) R(x, y)}
\end{aligned}
$$


for $x \in(0, K]$ and $y=\psi(x) \leq \hat{y}$ and $\sigma^{\prime}(x)=0$ for $\psi(x) \geq \hat{y}$. By the assumption $H R>-N Q$, we have

$$
-1<\sigma^{\prime}(x)<0 \quad \text { for } x \in(0, K] .
$$

Consequently, Corollary 4.5 yields the global attractivity. By Theorem 5.2 in [11], the positive equilibrium is clearly locally stable, proving the theorem.

If $m=1$, we need the following assumption to ensure the existence of a positive equilibrium, namely

$$
p(k)>\frac{s}{c}
$$

For simplicity, we also assume the following.

(H5): $y=\psi(x)$ can be solved from the equation

$$
s+q(y)=c p(x)
$$

for all $x \in[\tilde{K},+\infty)$ for some $\tilde{K}<K$.

THEOREM 5.2. Suppose $m=1$ in system (5.1). Assume $H R>-N Q$ for all $0<$ $y<\left|g(0) / p^{\prime}(0)\right|$ and $0 \leq x \leq K$. If (5.8) and (H5) are satisfied, then there exists a unique positive equilibrium for system (5.1) which is globally asymptotically stable.

PROOF. Let $\tilde{K} \geq 0$ be the smallest value such that $y=\psi(x) \geq 0$ can be solved from (5.9) for $x \in[\tilde{K},+\infty)$. It is easy to see that $y(\tilde{K})=0$. Now, we define $y=\psi(x)=0$ for $x \in[0, \tilde{K}$ ) (also see Remark 5.3). Similar to the proof of Theorem 5.1 , we can show the result.

\section{Discussion}

In this paper we have considered a predator-prey system with time delay due to gestation and have derived criteria for uniform persistence and for global attractivity of a positive equilibrium.

The results in this paper may by some careful analysis be extended in several ways. First the same results as in Theorem 4.2 would hold if $y(t)$ in the first equation of (2.1) is replaced by $y\left(t-\tau_{1}\right)$. This may be of mathematical interest although its biological significance is unclear.

Secondly, results similar to Theorem 4.2 should be valid in the case that $\tau$ is state dependent, that is, $\tau=\tau(y(t))$ or $\tau=\tau(x(t))$ provided $\tau(\cdot)$ is locally Lipschitzian. We would also require a Lipschitzian intitial function in (2.2). 
The critical assumption (H4) required for the global attractivity is somewhat restrictive. It would be of interest if one could replace ( $\mathrm{H} 4)$ with a less restrictive assumption allowing for a prey isocline which both increases and descreases as was done in [17] in the ODE case.

Finally, it would be of interest if our results could be extended to food chains.

\section{Acknowledgements}

The first author began research while visiting the University of Alberta with support by the Central Research Fund.

The second author had research partially supported by the Natural Sciences and Engineering Research Council of Canada, Grant Number NSERC A4923. Research completed while visiting the Centre for Dynamical Systems and Nonlinear Studies and School of Mathematics, Georgia Institute of Technology, Atlanta, GA.

\section{References}

[1] G. J. Butler and P. Waltman, "Uniformly persistent systems", Proc. Amer. Math. Soc. 96 (1986) 425-430.

[2] G. J. Butler, H. I. Freedman and P. Waltman, "Persistence in dynamical systems", J. Differential Equations 63 (1986) 255-263.

[3] Y. Cao, J.-P Fan and T. C. Gard, "Uniform persistence for population interaction models with time delay", Appl. Anal. 51 (1993) 197-210.

[4] Y. Cao and T. C. Gard, "Extinction in predator-prey models with time delay", Math. Biosci 118 (1993) 197-210.

[5] Y. Cao and T. C. Gard, "Uniform persistence for population models with time delay using multiple Lyapunov functions", J. Diff. Integr. Equations 6 (1993) 883-898.

[6] Y. Cao and T. C. Gard, "Ultimate bounds and global asymptotic stability for differential delay equations", Rocky Mountain J. Math 25 (1995) 119-131.

[7] K.-S. Cheng, S.-B Hsu and S.-S. Lin, "Some results on global stability of a predator-prey system", J. Math. Biol. 12 (1981) 115-126.

[8] H. I. Freedman, Deterministic Mathematical Models in Population Ecology (Marcel Dekker, New York, 1980).

[9] H. I. Freedman and K. Gopalsamy, "Global stability in time-delayed single species dynamics", Bull. Math. Biol. 48 (1986) 485-492.

[10] H. I. Freedman and P. Moson, "Persistence definitions and their connections", Proc. Amer. Math. Soc. 109 (1990) 1025-1033.

[11] H. I. Freedman and V. S. H. Rao, "The tradeoff between mutual interference and time lags in predator-prey systems", Bull. Math. Biol. 45 (1983) 991-1004.

[12] H. I. Freedman and S. Ruan, "Uniform persistence in functional differential equations", J. Differential Equations 115 (1995) 173-192.

[13] H. I. Freedman and P. Waltman, "Persistence in models of three interacting predator-prey populations", Math. Biosci. 68 (1984) 213-231. 
[14] K. Goopalsamy, "Time lags and global stability in two species competition", Bull. Math. Biol. 42 (1980) 729-737.

[15] J. K. Hale, Theory of Functional Differential Equations (Springer Verlag, New York, 1977).

[16] J. K. Hale and P. Waltman, "Persistence in infinite dimensional systems", SIAM J. Math. Anal. 20 (1989) 388-395.

[17] S.-B Hsu, "On global stability of a predator-prey system", Math. Biosci 39 (1978) 1-10.

[18] Y. Kuang, Delay Differential Equations with Applications in Population Dynamics (Academic Press, Boston, 1993).

[19] L. P. Liou and K.-S Cheng, "Global stability of a predator-prey system", J. Math. Biol. 26 (1988) $65-71$.

[20] W. Wendi and M. Zhien, "Harmless delays for uniform persistence", J. Math. Anal. Appl. 158 (1991) 256-268. 